\title{
Peristomal Dermatology
}

\author{
Bonnie Alvey, R.N. ${ }^{1}$ and David E. Beck, M.D. ${ }^{2}$
}

\begin{abstract}
Inflammatory or infectious conditions affecting the skin around an intestinal stoma are common and may be a source of considerable aggravation to patients. Recognition and prompt appropriate treatment of these conditions improves their quality of life. Peristomal pyoderma gangrenosum represents a most unusual peristomal skin condition that is difficult to treat; resolution often requires diagnosis and therapy of underlying inflammatory bowel disease.
\end{abstract}

KEYWORDS: Intestinal stomas, dermatology, pyoderma gangrenosum

Objectives: At the completion of this article, the reader should be able to recognize and treat common peristomal skin conditions and pyoderma gangrenosum.

Every year thousands of people undergo surgical procedures for diversion resulting in an intestinal or urological stoma. Advances in ostomy equipment/ pouches and wound care options, as well as access to wound ostomy continence (WOC) nursing expertise have led to better outcomes for this patient population. However, this progress has been hindered by today's shorter hospital stays and laparoscopic procedures, which result in less time for patients to learn ostomy basics and measures to prevent skin complications. The surgeon's goal is a well-constructed stoma. Unfortunately, this goal is not always achieved, as 10 to $70 \%$ of ostomy patients experience some type of peristomal skin problems. ${ }^{1}$ Many of these issues can be prevented when an enterostomal therapy (ET) or WOC nurse is an integral part of patients' preoperative and postoperative care. However, as some colorectal surgeons and consequently their patients do not have access to a WOC nurse, it is important for the surgeon be able to identify and manage any peristomal complications that may occur.

Thousands of patients suffer from some type of peristomal skin disturbance at some point while living with an ostomy whether temporary or permanent. There are mechanical, chemical, microbial culprits to these skin complications. These dermatological conditions can range from an abrasion or skin stripping to the more complex, pyoderma gangrenosum. In this article, we will address the most common peristomal skin complications and their treatment options.

\section{MECHANICAL TRAUMA}

The most common causes of dermatitis is an ill-fitting appliance accompanied with mechanical stripping. ${ }^{2}$ Frequent pouch changes can lead to epidermal damage or mechanical stripping that leaves the skin painful, moist, and possibly bleeding.

Cutting pouch appliances too large exposes the peristomal skin to ileostomy output, which is rich in enzymes. The exposed stratum corneum becomes scalded or excoriated. Excoriated skin is defined as that which has been traumatized, worn away, or abraded, often in the presence of maceration due to incontinence. ${ }^{3}$ The distribution of excoriation provides clues to the
${ }^{1}$ Ochsner Clinic Foundation Hospital, ${ }^{2}$ Department of Colon and Rectal Surgery, Ochsner Clinic Foundation, New Orleans, Louisiana. Address for correspondence and reprint requests: David E. Beck, M.D., Department of Colon and Rectal Surgery, Ochsner Clinic Foundation, 1514 Jefferson Hwy., New Orleans, LA 70121 (e-mail: dbeckmd@aol.com).
Stomas and Wound Management; Guest Editor, David E. Beck, M.D. Clin Colon Rectal Surg 2008;21:41-44. Copyright (C) 2008 by Thieme Medical Publishers, Inc., 333 Seventh Avenue, New York, NY 10001, USA. Tel: +1(212) 584-4662.

DOI 10.1055/s-2008-1055320. ISSN 1531-0043. 
practitioner as to whether the cause is an ill-fitting appliance or possibly an allergy to the appliance. ${ }^{4} \mathrm{~A}$ flush or retracted stoma presents a challenge and usually leads to excoriation or denuded skin. Once the skin has become denuded it is then much harder to achieve a good seal. To manage this problem, first assess the stoma characteristics and type of pouch or appliance being used. Patients are often wearing the same type of pouch placed while in the hospital and this may not be as appropriate once swelling has subsided. Often a pouch with convexity (an outward curve on backside of wafer) is indicated. Once the skin is healed a reassessment may indicate a convex wafer shape to be the best pouch option.

To treat the denuded skin, there are several choices. A no-sting liquid barrier/skin sealant can be applied, which provides a protective film and helps dry the area so that a pouch can adhere successfully. Skin sealant also reduces epidermal stripping and many people will incorporate this into their pouching routine. Sometimes, a light dusting of stoma powder can dry the skin enough to provide an adequate pouching surface. Many patients also report success with something as simple as a light coating of calamine lotion, another well-known drying agent. The most important course of action is to determine if the pouch in use is the best for that stoma and for that patient's contours. Fix the cause and the problems will resolve.

\section{Pressure Ulcers}

Another mechanical cause of peristomal complications is pressure on the skin by belts or convex flanges, especially in the presence of peristomal hernias. Some pressure sores may progress to full-thickness damage. In addition to reevaluating the patient for alternate appliances, the practitioner must also provide topical care to promote healing of the ulceration. This can be challenging as most people wearing convexity need the convexity to maintain an adequate seal. ${ }^{5}$

\section{Mucocutaneous Separation}

Mucocutaneous separation can occur in the early postoperative period. This does not usually necessitate surgical intervention, but does require wound care interventions along with pouching. A common and effective treatment is to fill the defect left from the separation with stoma powder and then use stoma paste to "caulk" the open defect.

\section{IRRITANT DERMATITIS}

Peristomal dermatitis is most often caused by stool or urine that is an irritant to the skin. The amount of exposure as well as character of effluent will determine extent of peristomal irritation. Stool leaking on the skin causes inflammation. The extent of this irritant dermatitis can vary with small bowel output being caustic enough to produce skin erosions. The prevention of any dermatitis is the primary focus in the early days and weeks after a new ostomy formation. Any degree of dermatitis will inhibit pouch adhesion, which then leads to more leakage and skin inflammation. Consequently, a painful dermatitis develops that is challenging to manage. This can be particularly distressing to a new patient as he or she adjusts to the presence of a stoma as well as their disease process. Prevention of this dermatitis leads to a much better outcome and a happier patient.

\section{INFECTIOUS DERMATITIS}

\section{Fungal}

Another common cause of peristomal dermatitis is Candida. Candida albicans is the most common species found on skin (66\%). ${ }^{6}$ The peristomal skin is an ideal environment for yeast proliferation as the skin is warm, dark, and any leakage or immunocompromised condition will contribute to this complication. Peristomal candidiasis has been reported with both urinary and fecal diversions. ${ }^{7}$

Cutaneous candiditis presents as reddened, shiny patches with satellite papules and pustules, regular or irregular borders (Fig. 1). Based on a Cochrane review, the use of azoles such as miconazole and clotrimazole are best first-line treatments. These are cost effective, accessible, and do not require a prescription. The allylamines, such as Lamisil $^{\circledR}$ (Novartis Pharmaceuticals Corp., East Hanover, NJ) are suggested for patients who fail or are resistant to over-the-counter agents. Drugs in this category generally are more costly. ${ }^{8}$ Antifungal powder can be applied to skin, rubbed in gently, and followed by a skin sealant. Once the sealant is dry the pouch can be placed.

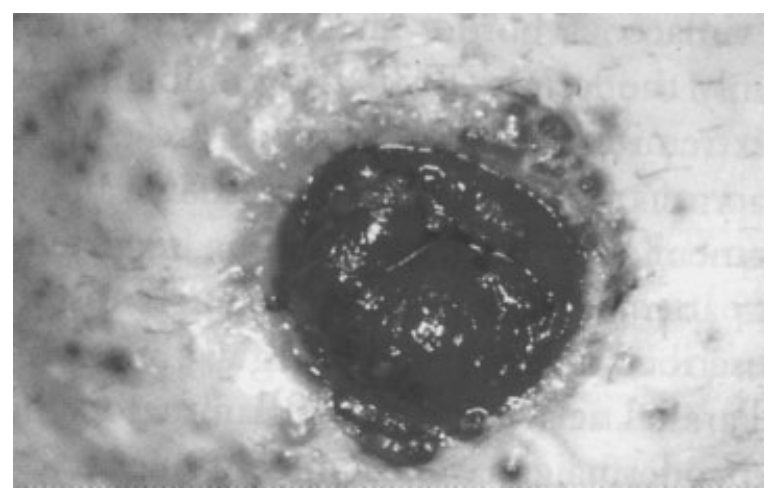

Figure 1 Peristomal candidiasis. 


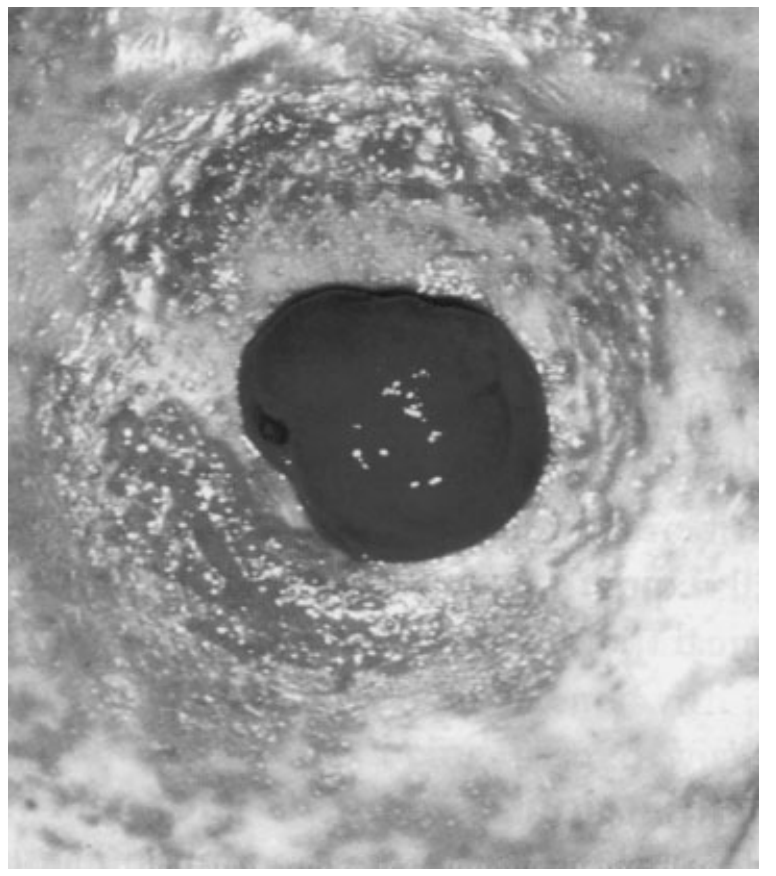

Figure 2 Folliculitis.

\section{Folliculitis}

Hair follicle inflammation (folliculitis) can cause the skin to become irritated, especially in the person who must shave routinely to maintain a seal. It also occurs in those who do not shave as removal of the pouch adhesive can cause trauma leading to folliculitis. Staph aureus is the most frequent microbial culprit leaving an erythematous and pustular area that resembles candidiasis (Fig. 2). If antifungal treatment does not clear the problem, it is likely to be bacterial and an antibacterial powder is indicated.

\section{ALLERGIC REACTIONS}

Allergic reactions causing dermatitis are less common with modern adhesives. A potential allergen provokes a response and antibody production, which on reintroduction triggers a response. The inflammation of the skin usually mirrors the pattern of the allergen (Fig. 3). A frequent allergen is the pouching system adhesive. Patch testing will often identify the offending agent. ${ }^{8}$ As there are many choices of pouching systems, one can usually find an alternative that will be tolerated. A topical steroid preparation may reduce allergic reactions while alternative systems are tried, but this may interfere with adhesion of the appliance.

\section{PYODERMA GANGRENOSUM}

One of the less common but more challenging issues is ulceration of the skin or pyoderma gangrenosum (PG). PG was first described in 1930 by Brunsting and colleagues.9 Although PG was initially thought to be

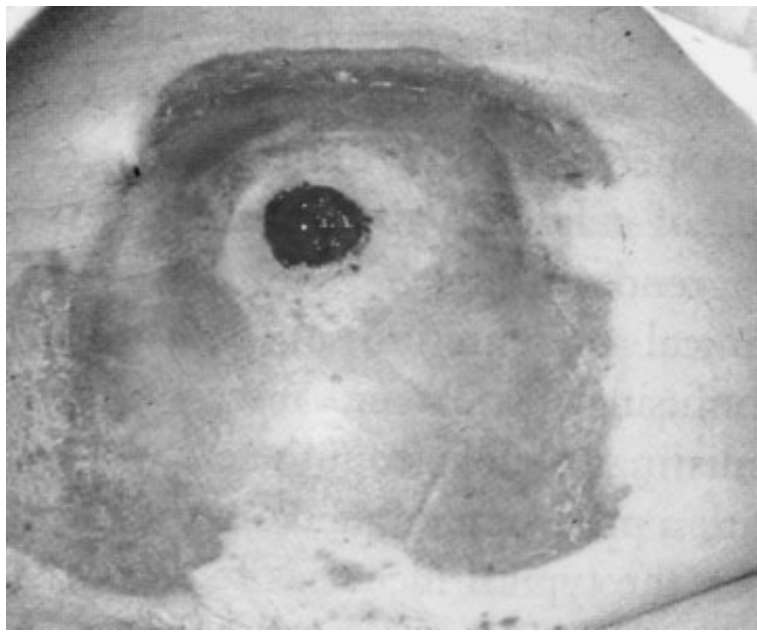

Figure 3 Cutaneous allergic reaction to pouch.

associated with Crohn's or inflammatory bowel disease, it is now also known to be associated with malignancies, blood dyscrasias, diabetes, and hepatitis. ${ }^{10}$ Pyoderma has been described in several forms, but the ulcerative presentation usually occurs on the abdomen, perineum, and lower extremities. The lesions begin as discrete pustules that erupt and coalesce into a classic painful ulcer with a violaceous border and undermined edge (Fig. 4). Multiple lesions are common.

Colwell has noted that PG is a significant complication associated with prolonged pain and increased morbidity. ${ }^{11}$ As its etiology and pathophysiology are poorly understood, multiple treatments have been employed. These include multiple topical therapies and corticosteroids given topically, intralesionally, or orally. Cyclosporin has also been tried, but Fauld and associates

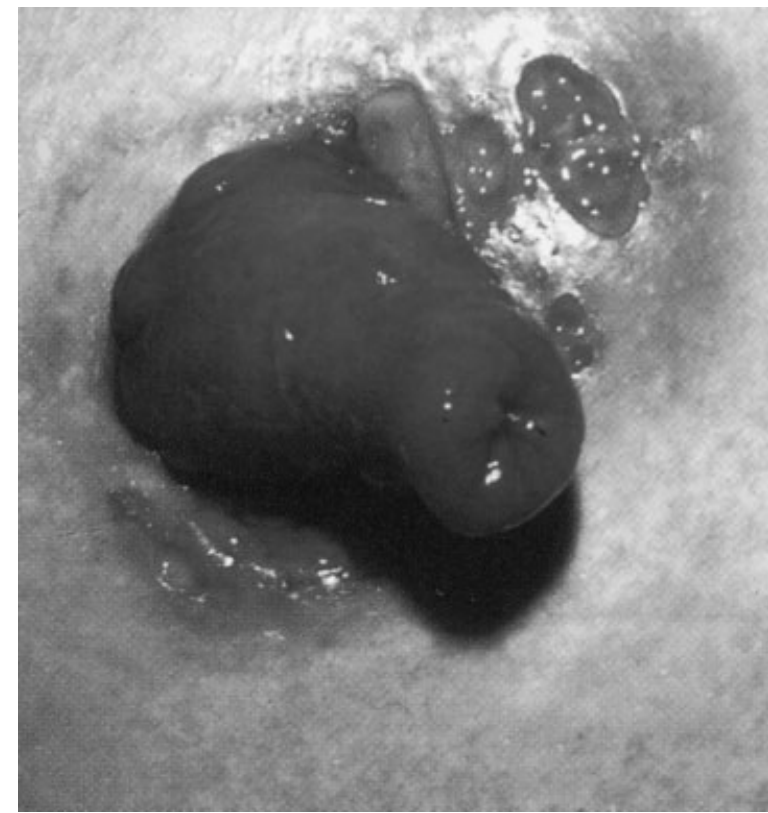

Figure 4 Pyoderma gangrenosum above the stoma. 
noted weak evidence for its use in pyoderma gangrenosum. ${ }^{12}$

Peristomal pyoderma presents challenges to successful pouching. The size of the ulceration and its proximity to the stoma affects the seal of the appliance to the skin. The goal for management is to promote healing while maintaining adequate wear time of the pouch. The painful nature of PG ulcerations influences the options for topical care. Small ulcerations can usually be treated with stoma powder or antimicrobial powder covered by a piece of hydrocolloid. A foam dressing over the ulceration is helpful if the ulcer is particularly moist. Silver dressings in sheet form or calcium alginates have also been effective. The goal of topical therapy is to use a modality that can absorb the moisture and allow for appliance adhesion.

\section{SUMMARY}

It is important that the WOC nurse be involved with these patients for preoperative stoma siting as well as through the postoperative course. Monitoring complications and providing early intervention with skin complications will result in better outcomes. ${ }^{13}$

\section{REFERENCES}

1. Colwell J, Goldberg M, Carmel J. The state of the standard diversion. J Wound Ostomy Continence Nurs 2001;28: 6-17
2. Erwin-Toth P. Prevention and management of peristomal skin complications. Adv Skin Wound Care 2001;14:1-6

3. Collins F, Hampton S, White R. A-Z Dictionary of Wound Care. London: Quay Books; 2002

4. Lyon CC, Smith AJ, Griffiths CEM, Beck MH. The spectrum of skin disorders in abdominal stoma patients. Br J Dermatol 2000;143(6):1248-1260

5. Hampton B. Peristomal and stomal complications. In: Hampton B, Bryant R, eds. Ostomies and Continent Diversions: Nursing Management. St Louis, MO: Mosby; 1992:108

6. Evans EC, Gray M. What interventions are effective for the prevention and treatment of cutaneous candidiasis? J Wound Ostomy Continence Nurs 2003;30(1):11-16

7. Ratliff CR, Donavan AM. Frequency of peristomal complications. Ostomy Wound Manage 2001;47:26-29

8. Crawford F, Hart R, Bell-Syer S, Torgerson D, Young P, Russell I. Topical treatments for fungal infections of the skin and nails of the foot. Cochrane Database System Review 2002;(2):CD001434

9. Brusting LA, Goeckerman WH, O'Leary PA. Pyoderma gangrenosum:Clinical and experimental observations in 5 cases occurring in adults. Arch Dermatol 1930;22:655-680

10. Crowson AN, Mihm MC, Magro C. Pyoderma gangrenosum: a review. J Cutaneous Pathol 2003 30:97-107

11. Colwell JC. Stomal and peristomal complications. In: Colwell JC, Goldberg MT, Carmel JE, eds. Fecal and Urinary Diversions: Management Principles. St Louis, MO: Mosby; 2004:308-325

12. Faulds D, Goa KL, Benfield P. Cyclosporin. A review of its pharmacodynamic and pharmacokinetic properties, and therapeutic use in immunoregulatory disorders. Drugs 1993; 45:953-1040

13. Loehner D, Casey K, Schoetz DJ Jr. Peristomal dermatology. Clin Colon Rectal Surg 2002;15:209-214 